\title{
Nuevas observaciones de Corioepitelioma
}

\author{
Por el doctor Fernando Mera González \\ Profesor Encargado de Obstetricia. Hospital de San Juan de Dios.
}

En los primeros meses del año pasado publicamos en esta misma revista la historia clínica de una enferma que padeció de Corioepitclioma después de un cmbarazo a término, y no sabemos si por coincidencia o acaso por tener presente en la memoria el caso que motivó su muerte, pudimos a fines del mismo ano establecer nuevamente el diagnóstico de Corioepitelioma en una paciente que ingresó al servicio de maternidad y a principios del presente volvimos a confirmarlo en otra enferma con sintomatología parecida, en el mismo servicio.

En las historias clínicas referidas se comprobi por los resultados anatomo patológicos y biológicos el diagnóstico clínico inicial de degeneración epiteliocorial. Antes de describir la sintomatología predominante en ambas pacientes, queremos llamar la atención de los (inecólogos y Obstetras sobre estos puntos: Primero: la frecuencia de dicha neoformación es alta entre nosotros, si se tiene en cuenta que en los dos años en que desempenamos la Jefatura de la Clínica en el Instituto de Proteción Matcrna e Infantil (Servicio del Profesor Rodrí. gue Aponte) observamos, en un total de 6.030 enfermas hospitalizadas, tres casos de degerreración maligna. Segundo: La colución clínica, los resultados anatomopatológicos y los scrológicos son las bases del diagnóstico, cuando ha mediado embarazo antiguo o reciente. En las tres experiencias encontramos una colución clinica acorde con la sintomatología clásica, eliminacion hormonal sostenida superior a las 500.000 unidades (Galli Mainini) y descripción anatomopatológicat demostrativa de la lesión. Tercero: Mayor ocurrencia de la degeneración maligna después de embarazo molar, sin que pueda descartarse que la hubicran sufrido las pacientes cuyas historias relataremos en seguida y en quienes no fue posible diagnosticarla por haberse efectuado sus abortos, de manera espontánea, fuera del Servicio. Cuarto: Necesidad del control periódico en la eliminación hormonal de gonadotropinas coriónicas por el tiempo mínimo de dos años en las pacientes que hayan sufrido de mola hidatiforme. La eliminacion de hormonas disminuye gradualmente hasta desaparecer, cuando hay mejoria total, y aumenta progresivamente cuando se inicia la degeneración maligna. Quinto: Conveniencia de enviar a examen histológico los restos embrionarios extraídos digital o instrumentalmente, después de un aborto tópico in completo, cuando existan adherencias anormales o características especiales du- 
dosas. Sexto: Conveniencia de practicar reacciones biolégicas cuantitativas de Galli Mainini o de Friedmann, para establecer precozmente la iniciación de la degeneración maligna corial, en todos aquellos casos de abortos incompletos con adherencia persistente de las vellosidades a la decidua materna, además de hemorragias discretas o profusas. Séptimo: Precocidad en el diagnístico para el éxito del tratamiento, pues la invasión de los tejides vecinos o distantes por el tumor proliferante y metastásico agrasa el pronostico: la primera de las enfer mas obscrvadas, hizo metastasis en el cerchro, ol higado y el pulmon: en la so gunda, después de la histerectomia total, se reprodujo la lesión en la vagina: ! solamente en la tercera se logro la mejoria total por haber sido sattisfactorios los controles clínicos y biologicos practicados durante los cuatro meses pesteriores a la operación, y Octavo: La extirpación del útero y de los anexos, cuando se hallen lesionados macroscópicamente, es el procedimiento más aconsejable.

Para llegar al diagnóstico es necesario tener siempre en cuenta la posibilidad de degeneración epitelio-corial y su posible presentación en todos aquellos casos de mujeres de actividad genital con antecedentes de embarazo, ya que la sintomatología clásica no se presta a confusién, como lo veremos en el relato de las diferentes historias clínicas.

Nombre: B. S. Edad: 22 años. Historia No. 1.730.

Diagnóstico clínico: Cormolprtelioma.

Diagnóstico de admisión: Fibroma uterino: Metropatia hemorrágica? Abor to incompleto?

Diagnóstico definitivo: Comoeprteloona. Metástasis vaginal.

Invasión de parametrios y de vejiga. Defunción.

Antecedentes hereditarios: Sin importancia.

Antecedentes personales: Tifo, sarampion.

Menarquía a los 15 años. Cielo 30/4, algemenorrea, leucorrea.

Scrologia: negatival.

Cuatro embarazos normales, con partos nomales a termino y fetos vivos.

Embarazo actual: Lltima menstruacion: septiembre, 1952. Con cefaleas y hemorragias.

Examen general: Temperatura: $37^{\circ}$ C. Piel sana. Senos normales. Esque leto normal. Pared abdominal flacida. Corazón normal. Pulso 110. Presión arterial: $120 \times 70$. Pulmones normales. Dientes incompletos y en mal estado. Lengua lavada, saburral.

Enfermedad actual: En lebrero de 1952, en el Servicio de Gincología de San Juan de Dios, le fue practicado un raspado uterino, por aborto incompleto según lo relata la enferma. Desde ese tiempo continuó con hemorragias genita les dolorosas, que se suspenden en ocasiones por tres dias máximo.

Al llegar al Servicio de Maternidad se observa mareado estado anémico, con un estado general precario y no sangra por la vía genital. El ciclo mens trual antes del "aborto de febrero" era de 30/4. El tacto vaginal, que se le hizo el 14 de julio de 1952 revela: vulva, vagina y perineo normales, aunque muy pálidos; cuello central, cerrado; el cuerpo del útero aumentado de tamaño como 
*orrespondiente a tres meses de embarazo; se encuemar en anteversollexion exagerada y es de consistencia dura y doloroso. Los fondos de saco vaginales están libres.

Hospitalizada la enferma, tuvo temperatura oscilante hasta de 40 grados, per los cuales se prescriben antibiéticos, lo yue nomalizo la temperatura despuces del tratamiconto.

1: 19 de julio es examinada la enferma nuvamente por personal del Ser.

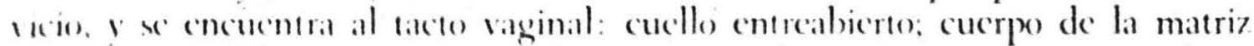
.umentade de tamano: a trases del orilicio cervical. se aprecia una masa dura. pediculadia, you da la impresion de un póliper. Exe dia le practicaron un legra. do uterino, bajo anestesia general con éter. Se tapono la cavidad.

El producto del legrado dio el siguiente resultado anatomopatológico:

"Descripción Macroscóplea: Varios fragmentos de tejido de consistencia blanda, de color rosado oscuro, el mayor de hos cuales mide $2.7 \times 2.5 \times 1.6$ centímetros.

\section{Diagnóstico Microscópico: Probable emplarazo.}

Los cortes muestran coágulos de sangre dạntro de los cuales se aprecian cscasas formas de estructura, que dan la impresión de ser vellosidades placentarias. Hay masas compactas de leucocitos con marcado predominio de los polimucleares."

La paciente continuó sangrando después de retirar la mecha-tapón yue se le colocó, y a pesar del tratamiento útero-ténico establecido.

En estas condiciones la examinamos por primera vez y ordenamos inmediatamente exámenes scrológicos de Galli Mainini, por presumir una degeneracion epitclio-corial.

El 30 de julio se titularon las gonadotropinas corionicas dando por resultado una eliminación hormonal de 550.000 unidades por litro de orina.

Por la sinfomatología clínica apreciable y por el resultado serológico, era indiseutible establecer ef diagnostico de coriópitelioma, y en estas condiciones se intervino quirurgicamente d dia 31 de julio para practicar una histerectomía fotal con reseccion de anexos ya yue se hallaron macrosiopicamente lesionados. Se anota, como detalle especial del acto quirurgico, el hecho de hallarse la superlicic uterina con peyueñas vesiculas llenas de un líquido claro.

El postoperatorio fue normal y se preseribio tratamiento a base de antibioticos, analgésicos y antianémicos generales.

El 2 de agesto, la enferma experimenta una nueva hemorragia vaginal escasa que persistio hasta el 10 de agosto.

1:l dia 6 de agosto, una nucva titulación hormonal (Galli Mainini) da por resultado una eliminación de 250.000 unidades por litro de orina.

El estado general da la impresion de una mejoria franca, a pesar del cua (ro termico sublebril.

El 8 de agesto, en vista de que continuaba la hemorragia genital, se prac ticó nucvo examen vaginal apreciándose una peguena masa necrótica hemorrá gica, que posteriormente aumentó de volumen hasta ocupar totalmente el ves. tíbulo vaginal, con diámetros aproximados de $3 \times 4 \mathrm{cms}$. de longitud. 
Las piezas obtenidas en la histerectomía se enviaron al laboratorio de anatomía patológica, de donde respondieron:

"Descripción Macroscópica: (útero. Histerectomía total con salpingoooforectomía bilateral) que pe 250 gramos y mide 11.2 por 8.7 por $4.8 \mathrm{cms}$. Longitud de la cavidad uterina: $9.5 \mathrm{cms}$. Espesor del miometrio $2.1 \mathrm{cms}$ y del endometrio $0.2 \mathrm{cms}$.

En la mucosa y hacia de fondo se encuentra una masa de tejido exulerante que mide $3.5 \mathrm{cms}$ de diámetro, cuyal superticie de seccion tiene aspecto de ne crosis. Las trompas miden por término medio 7.8 porr 0.6 por 0.5 cms. No presentan nada especial. Los ovarios pesian en conjunto $9 \mathrm{grs}$ y miden por término medio 3.4 por 2.5 y por $0.9 \mathrm{cms}$. Macroscipicamente son de aspecto normal.

Diagéóstico Microscópico: Coriocpitelioma. Cervicitis sub-aguda.

En el cuello uterino hay un proceso inflamatorio que afecta especialmente la capa uterina. Los cambios de estructura consisten en edema, focos de necrosis con formación de pus, dilatación de los vasos sanguíneos con fenómenos de diapedesis e infiltración por leucocitos poli y mononucleares. En el cucrpo, la capa interna presenta un neoplasma maligno moderadamente infiltrante que se acompana de grandes áreas de necrosis, con formación de pus y cuya estructura recuerda la del tejido placentario. Los elementos que lo forman son muy ricos en cromatina, presentan diferencias de tamano $y$ de forma $y$ dejan ver figuras de división indirecta. En los tubos y ovarios, no se encontró nada especial.

\section{(Fdo.) M. Sánchez Herrera."}

El 23 de agosto, en vista de la metástasis vaginal, se traslada al servicio de aislamiento porque la paciente se negé a tratamiento en el Instituto de Radium.

En el servicio de aislamiento se encuentra al examen lo siguiente: Hiper trofia con necrosis de la mucosia vaginal. se aprecia un tumor exulderante del fondo vaginal, singrante, con leucorrea abundante y doloroso.

El estado de la paciente es regular, con marcado cestade anémico, hay cons tipación intestinal y trastornes de la micción. Temperatura $37^{\circ}$ C., pulso 84 , presión arterial $70 \times 50$. Se le prescribe medicacion antianćmica.

El t de septicmbre hay mayor crecimiento de la neolormación vaginal. con mal estado general y gran palides por anemia. Hay hemorragia vaginal abundante. Incontinencia parcial de orina.

El resultado de Galli-Mainini es de 1.000 .000 de unidades por litro de orina.

La enferma continuó con hemorragias vaginales y se hizo más precario el estado de salud, hasta el dia 24 de octubre en que murió a las 7 de la mañana.

No se le practicó autopsia por impedirlo los familiares.

Hay los siguientes exámenes de laboratorio:

Análisis de: Sancine: Hematies 2.460.000 por mm. c. Leucocitos: 9.600. Polimorfonucleares neutrofilos $60 \%$. Eosinofilos: 1\%. Linfocitos pequeños $35 \%$. Formas de paso: $4 \%$.

Análisis Parcial de Orina: Reacción: ácida. Albúmina: negativo. Glycosa: negativo. Sedimento: células, $x x$; leucocitos, $x$. 
Análisis de Sangre: Hematíes, 2.020 .000 por $\mathrm{mm}$. c. Leucocitos $10.4(0)$ por mm. c. Hemoglobina: 4 grs. \%. Hematocrito $16 \%$. Polimorfonucleares neu trofiles, $78 \%$. Eosinofilos, 1\%. Grandes mononucleares, $4 \%$. Formas de paso, 5\%. Normoblastos, 1\%. Volumen globular medio: 59 micrones.

Al comentar esta historia quico hacer resiltar el peligro de confusión con simples alerter incompletes en los cases de corio cpitelioma, por la sintomatolo ghe p.urcida y por la mejoria relatio que experimentan después de someterlas .1 r.1eprede uterino. En este caso hube confusion con un pólipo uterino por su anducion clinica y por el aspecto de la neoformacion. El diagnostico de ingreso fue variado: Se pensi en una fibromatosis uterina, en una metropatia hemo rigica, y en un aborto incompleto. Solamente la titulacion de gonadotropinas attamente positivas, sirvió de pauta para establecer el diagnóstico verdadero.

HISTORIA No. 97.-Nombre: B. F. Edad 35 años. Nació en Pesca (Boyací). Ingresó en enero 15 de 1953.

Diagnéstico: Aborto incompleto? Neo uterino?

Diagnóstico definitivo: Corio-epitelioma post-aborto.

Antecedentes hereditarios y personales sin importancia.

Menarquía a los 15 años. Ciclo 30 8. Menorragias, dismenorrea, leucorrea.

Oho embarazos normales con partos normales a término y fetos vivos. In aborto espontánco de 3 meses en la cuarta gestación.

Lmbarazo actual: última menstruación: agosto 22 de 1952. Vómitos, maros, cofalea y fosfenos.

Examen general: Estado general, regular. Piel anémica. Senos caidos. Pa - red aldeminal flácida y depresible. Pulso 128. T. A. 110 x 85. Pulmones: nor makes. Dientes: en mal estado. Lengua saburral. Temperatura $37^{\circ} \mathrm{C}$.

Infermedad actual: Dice la enlerma que hace t2 dias tuvo un aborto es jemtanco seguido de una hemorragia vaginal discreta, sin dolores, y por esta causa se vio precisada a hospitalizarse.

Antes, y por la misma causa que enuncia, se internó en el hospital de So gamoso, donde le practicaron un legrado, a pesar de lo cual le continuaron las lemorragias vaginales.

La paciente no precisa las características de los restos embrionarios ex. pulsados.

Examen (enero 15 de 1953): Genitales externos normales. T. V. Cuello central, ligeramente largo, blando y edematoso. El cuerpo de la matriz está au mentado de tamano. A través del orificio cervical se aprecia tejido blando, fria ble y que sangra con facilidad. Los fondos de saco vaginales se encuentran libres.

Se ordena transfusión de sangre total y tratamiento antianémico.

Encro 18/53: T. V. Cuello uterino grueso y blando, permeable a un dedo, en el centro de la cúpula vaginal; a través del cuello y dentro de la cavidad uterina palpo tejidos blandos en un todo parecidos a restos embrionarios, que 
sangran con facilidad; el cuerpo del utero se encuentra aumentado de tamaño, como correspondiente a un embarazo de 2 meses y en fucrte anteverso-flexión. En estas condiciones se practico un legrado instrumental para enviar la muestra al patólogo.

Como d.110 curioso, durante el legrado, que se realizó con anestesia intra venosa, se hallo una fuerte e intima adherencia de los tejides al endometrios al miometrio. Se ordenaron reaceiones cuantitatiats de Cialli Mainini.

Enero 22: Comadetropinas corionicas positivin part 500.000 unidades por litro de orin:ı.

Febrero 3: Ricsultado Anatomo patolugico: Daignostico clinico: Degencra ción epitclionorial.

Diagnósico microscópico: "(omoma. Ll material enviado para examen muestra endometrio, pequeñas zonas de miometrio y un neoplasma de origen placentario, acompanado de necrosis y de reaccion inflamatoria. Se ven vellosidades placentarias, en las cuales hay proliferación tanto de sincicium como de las células de Langhans.

Hay áreas de hiperplasia difusa del neoplasma con alguna tendencia a in filtrar. Los elementos que forman el tumor son hipercromaticos, redondos y ovoides, de tamaro variado o tendientes a agruparse en masas cinciciales. Sólo ocasionalmente se observan formas de estructura, que dan la impresión de figuras de division indirecta. (Fdo.) M. Sánchez Herrera."

Con los datos del Laboratorio y de Anatomia Patolígica, a más del cuadro clínico, se establece en forma definitiva el diagnestico de corioepitelioma y se pocede a mejorar las condiciones de la enferma con transfusiones de sangre to tal para intervenir quirúrgicamente el dia 10 de febrero y realizar una histerec tomia total con extirpacion de ancexos.

El acto yuirúrgico se verifico sin ninguma complicación. El examen anato. mopatoligico de la picza extraida dio el siguiente resultado: "Utero y anexos.

Diagnistico clinion: coriocpitclioma.

Descripeion matcroscopical átero y anexes (histerectomia total con salpin go ooforectomia bilateral). El utero pesil aproximadamente 145 grs. (previamente abierto). Dimensiones: 12.3 por 7.8 por 4.2 cms.

la longitud de la cavidad uterina: $9.4 \mathrm{cms}$. Espesor del miometrio: $2 \mathrm{cms}$. y del endometrio 0.3 coms. El miometrio es de color gris amarillento, de consis tencia blanda; el endometrio es de aspecto congestivo hacia el fondo uterino y ligeramente mamelonado. El cuello uterino es de consistencia más blanda que el resto del órgano, edematoso y con un diámetro de $5 \mathrm{cms}$. Ia trompa izquierda mide 10 por 0.6 por 0.6 cms. y la derecha 10.4 por 0.5 por $0.5 \mathrm{cms}$. Al corte son permeables y no se observan alteraciones macroscópicas. El ovario derecho pesa 140 grs. y el izpuicrdo 39 grs.; estín recmplazados por una masa o racimo de quistes que van deste un tamano de $0.2 \mathrm{cms}$. hasta $7 \mathrm{cms}$ y contienen un líquido de color amarillo citrino, traslúcido en unos y viscoso y gelatinoso en otros, separados por tabiques de color gris nacarado.

Diagnóstico microscópico: Coriocarcinoma. Cervicitis crónica. Quiste múltiple del ovario. 
El material enviado para examen muestra útcro, lubos y ovarios. En el cuello del útero, el corium y las regiones periglandulares dejan ver hiperplasia moderada del tejiclo conjuntivo, edema c infiltracion leucocitaria mononuclear. En el cuerpo se encontro un neoplasma de origen placentario, que infiltra moderadamente al miometrio y yue se acompanal de necrosis con reacción infla-

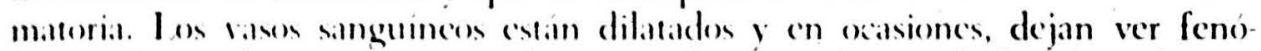
menos de tromberse yue comprometen total o parcialmente la luz. Los clemen. (1) nepplasticos unas veces recuerdan las colulas de Langhans, con hipercromarismo, diferencias de tamano y de forma y en ocasiones, liguras de división indirciti. Oeras veces se trata de masas cinciciales. En el tulo se encontró congestion. Las cavidades descritas anatomicamente en los ovarios son de origen folicular y están limitadas por epitclio cuboide estratificado, en ocasiones de citoplasma abundante y vacuolado. (Fdo.) M. Sinchez Herrera."

El post-operatorio de esta paciente transcurrió sin complicaciones. Se le practicó nueva titulación hormonal con resultado negativo, el día 22 de febrero de 1953.

Antes de darle de alta por curación, se le advirtió la necesidad de los controles biológicos durante un tiempo minimo de 2 años y en vista de que vive en Sogamoso y se le dificulta la venida a Bogotá, se le insinuó un control cada 2 meses para seguir cl estado de su salud.

La enferma salió el día 24 de febrero de 1953.

Mayo 26 de 1953: Clinicamente fue examinada y se encontrí en buenas condiciones. La reacción de (Balli-Mainini continúa negativa.

Esta última experiencia clínica comprucba plenamente dos cosas: La de generación epitelio-corial post aborto en nuestro medio y la facilidad de su diagnistico, basado en los exámenes anatomo patológicos de legrado y en la excre cion hormonal de gonadotropinas. Asimismo demuestra, por otra parte, la posibilidad de confusion con otras enfermedades alines, como d aborto incompleto 1) cl carcinoma del cuerpo uterino, que fucron los diagnósticos iniciales, cuando no se piensa previamente en la contidad patoligica que nos ocupa.

Aprovechamos la oportunidad para comentar algunas conductas obstétricas en relación con la mola hidatiforme, el aborto incompleto y el feto muerto in útero.

Establecido plenamente el hecho de degeneración coriocpitclial, no logramon explicarnos como especialistas avezados en las laleores obstétricas preconizan li conducta expectante en los cases comprobados de mola vesicular, y no fa vorcecen o ayudan la total evacuacion del contenido uterino por los diferentes inctodos conocidos, que no es del caso exponer en este estudio.

Bástenos recordar únicamente la similitud en la formación de los tejidos coriales de las pacientes alectadas de mola hidatiforme y de corio-epitelioma, como determinante de una conducta activa de rápido y total vaciamiento del contenido uterino. En tales extremos, vale más provocar el aborto molar por 
peligro de la degeneración maligna, que provocarlo por probables fenómenos anémicos, debidos a hemorragias escasas y repetidas, o profusas, o por temor a fenómenos tóxicos determinados por la transformación vesiculosa en evolución.

Desde luego, hay que advertir que, para farorecer el aborto molar, el obstetra debe estar seguro de la transformación vesicular de las vellosidades, como indicio vehemente de la alteracion, y requerir la colaboración del laboratorista, que dará luces acerca del aumento exagerado en la eliminación de gonadotro pinas. lgualmente, los rayos $X$ permitirán desaartar la presencia del feto cuando el tamaño del útero aparente un embaraze normal, y el estudio clinico de la entidad complementara la certeza en el diagnóstico.

En cuanto al aborto incompleto comprobado, no se justifica la contempo rización indefinida para lograr la expulsión total de los restos embrionarios con el concurso de los dilerentés ocitó icos conocidos, sino que, en nuestro concepto, se debe intervenir digital o instrumentalmente para obtener la total evacuación uterina. Con este sistema se evita la posible degeneración maligna de los restos embrionarios adherentes y activos así como las complicaciones frecuentes de los abortos incompletos, consistentes en hemorragias e infección.

Cuando el feto muere in úero, y se establece el diagnóstico cierto, conviene buscar su expulsión por los diferentes métodos obstétricos, ya que con cllo se evita la posible transformación maligna del tejido placentario o la provección de émbolos con tejidos epitelio-coriales en los diversos órganos de la economa, donde pueden sufrir la modificacion carcinomatosa. Es éste, a nues tro modo de ver, el mayor peligro de la muerte del feto dentro del útero, con las probables consecuencias de infección de los fetos macerados y la preocu pación permancente que entraña para la futura madre la conservación en su vientre de un feto sin vida.

\section{BIBLIOGRAFIA}

1.-Hernando Acosta Sarmiento. Conferencias de Obstetricia. 1948.

2.-M. Kirschner. Operaciones de los órganos genitales femeninos. Editorial Labor (Madrid, Barcelona, Buenos Aires, Rio), 1949.

3. Crossen \& Crossen. Ginecología. Utea, 1940.

4.-W. Stoeckel. Tratado de Obstetricia. Editorial Modesto Uson, Valencia, España, 1945 .

5. - Nubiola Zárate. Tratado de Obstetricia. Tomo II, Editorial Labor, 1951.

6.-D. Lee. Greenhill. Principios y Práctica de Obstetricia. Utea, 1945.

7. J. Moragués Bernat. Clinica Obstétrica.

8. - V. Conill Montobbio. Tratado de Ginecologia. Editorial Labor, 1946.

9.-Calatroni. Terapéutica Ginecológica. Editorial El Ateneo, 1946. 